\title{
REVISTAS CIENTÍFICAS E A CONSTITUIÇÃO DO CAMPO DE ESTUDOS DE GÊNERO: UM OLHAR DESDE AS "MARGENS"
}

\author{
MARIA MARGARET LOPES E ADRIANA PISCITELLI \\ Núcleo de Estudos de Gênero-PAGU/UNICAMP
}

\begin{abstract}
Resumo: Este artigo trata do papel das revistas científicas na construção do campo de estudos de gênero. A partir da sociologia da ciência, da história da ciência e da experiência das autoras na publicação dos CADERNOS PAGU, examina-se a interação desta com a agenda feminista. Busca-se discutir o efeito das tensões e "negociações" na publicação de revistas científicas, além da constituição do campo de estudos de gênero. As ações políticas, as tensões, as negociações, o processo de "peer-review", o "controle" do conhecimento científico e as normas editoriais são analisadas no quadro das publicações cientificas - a formação de um campo de pesquisa e a reprodução de centralidade/periferia no Brasil. Finalmente, aponta-se para questóes relativas ao aumento da visibilidade da Revista, da diversificação do seu conteúdo e da cooperação com pesquisadores latino-americanos e de outras partes do mundo.

Palavras-chaves: estudos de gênero, história da ciência, revistas científicas, feminismo.
\end{abstract}

\section{Apresentação'}

A literatura voltada para uma leitura crítica da história da ciência e da política científica tem iluminado aspectos ligados à relevância das revistas científicas, já há algumas décadas. Essa importância é sintetizada numa frase, taxativa, com a qual, no final da década de 1980, a antropóloga argentina Hebe Vessuri iniciava um texto marcante nos estudos sobre a produção dos países periféricos:

\begin{abstract}
La investigación científica que no está publicada no existe. La publicación en una revista de prestigio reconocido asegura la prioridad en la producción de un resultado, acrecienta el crédito académico de un científico, legitima su actividad y permite la existencia de sistemas e comunicación científica ligados a procesos activos de persuasión, negociación, refutación y modificación, a través de los cuales el significado de las observaciones científicas al igual que de las interpretaciones teóricas tiende a ser selectivamente construido y reconstruido en el campo científico. ${ }^{2}$
\end{abstract}

Com essas palavras, a autora sintetizava o valor concedido às publicações acadêmicas nos estudos sobre política científica. Nesse trabalho chamava a atenção para a prática de utilizar indicadores internacionais na avaliação da produção científica 
quando incorporando as especificidades dos países não centrais, discutia o papel essencial que as publicações científicas desempenham na constituição dos campos disciplinares. Esse valor está vinculado, sobretudo, à centralidade outorgada a essas publicações em termos da constituição de campos de conhecimento.

Considerando algumas dessas questões e tomando como referência a revista de cujo comitê editorial as autoras participam, os Cadernos Pagu, este texto apresenta, sem qualquer pretensão de ser exaustivo, alguns comentários sobre o papel das revistas acadêmicas na constituição do campo dos estudos de gênero, no Brasil. Levando em conta as tensões específicas que afetam as publicações dos países considerados "não centrais", em termos de produção de conhecimento, considerar-se-á, em primeiro lugar, a maneira como, no marco dessas tensões, as revistas informadas por interesses políticos feministas estabelecem "negociações" entre seus objetivos e as pressões às quais estão submetidas. Em segundo lugar, o texto apresenta comentários sobre os efeitos dessas negociações na constituição do campo dos estudos de gênero no país.

\section{As revistas científicas "nas margens"}

A produção sobre publicações científicas converge em assinalar que a publicação de um artigo em uma revista indexada de prestígio nacional ou (de preferência) internacional outorga reconhecimento acadêmico aos autores, legitima sua atividade acadêmica e, fundamentalmente, sustenta a comunicação inter e intrapares em que se baseia o sistema social da ciência. De acordo com Lea Velho ${ }^{3}$, esse é, aliás, o motivo pelo qual geralmente se aceita como uma das normas mais fundamentais da ciência que o pesquisador deve divulgar seus resultados de pesquisa. Pois, a publicação de um resultado de pesquisa, ao ser referendado por seus pares, através de um complexo processo de negociação para se obter consenso, transforma-se em ciência sancionada, em "verdade científica".

Pressupondo a ciência como uma atividade essencialmente comunicativa, do ponto de vista da sociologia da ciência, aqueles que não submetem seus resultados a este escrutínio requerido pela publicação, não poderiam ser chamados de cientistas, dado que o ato de publicar é parte constituinte do conceito de pesquisador. As publicações possibilitam a existência de sistemas de comunicação, vinculados a processos ativos de persuasão, negociação, contestação e modificação, através dos quais observações e interpretações teóricas tendem a ser seletivamente construídas e re-construídas no campo científico ${ }^{4}$. Os periódicos científicos são percebidos, portanto, como os veículos essenciais da comunicação acadêmica. Isso justifica a centralidade que se thes concede na construção de modelos de indicadores de avaliação de desempenho científico ${ }^{5}$. A relevância concedida às publicações científicas, nas análises de desempenho é evidente no Brasil, dado o interesse local de participação nos processos internacionais dos sistemas de indicadores de Ciência e Tecnologia - C \& T.

Entre as nações latino-americanas, o Brasil é considerado bastante ativo no âmbito da construção e análise de indicadores científicos para subsídios de políticas científicas e tecnológicas, e, portanto, também em desenvolver políticas de avaliação de revistas científicas. Nesse sentido, embora tenham se iniciado antes por iniciativa do CNPq, diversos autores apresentam os estudos da FINEP dos anos de 1980 (Programa Setorial de Publicações em Ciência e Tecnologia) como marcos institucionais referenciais para tais estudos de forma mais sistemática no país e na América Latina ${ }^{6}$.

A importância outorgada às publicações no sistema social da ciência obriga atualmente, a que qualquer estratégia de análise de tais publicações leve em conta os estudos quantitativos e, evidentemente, suas críticas. Os indicadores quantitativos de análise 
de publicações científicas - reveladores de aspectos fundamentais dos processos de funcionamento das comunidades ou arenas científicas (se preferirmos os termos de Karen Knor-Cetina) só ganham sentido se contextualizados e articulados em análises de conteúdo que indicam tendências e explicitam escolhas nas negociações que se estabelecem nas constituições e consolidações de campos disciplinares e interdisciplinares.

Nos países não centrais, o problema das publicações acadêmicas é considerado um dos desafios cruciais para o desenvolvimento da capacidade científica nacional e regional. As revistas, que garantem prioridades de temáticas e abordagens, constituem condição indispensável para a efetividade da comunidade como um todo. São percebidas como elementos-chave no estabelecimento de sistemas de pesquisa independentes. Supõe-se que a colaboração entre os cientistas de um país e de uma região, como, por exemplo, a latino-americana, pode viabilizar o desenvolvimento de massas críticas em campos específicos, contribuindo para a criação de redes mais autocentradas que valorizem suas próprias pautas investigativas ${ }^{7}$.

No entanto, mesmo que as revistas acadêmicas criadas fora dos países centrais tentem gerar um sistema de comunicação local paralelo, que permita inclusive a participação no âmbito internacional, com o suporte oferecido pela inserção em uma comunidade científica - local ou regional -, dinâmica e criativa, é inegável a posição desvantajosa na estratificação internacional dessas revistas ${ }^{8}$. Isto é evidente quando se observa que, se nessas publicações há um confronto com a pesquisa internacional, o material nacional nelas publicado não recebe respostas equivalentes por parte dos pesquisadores estrangeiros, estabelecendo uma ausência de feed-back. Esse não reconhecimento pelos cientistas estrangeiros está vinculado a uma série de fatores políticoculturais e, inclusive, lingüísticos. Por exemplo, enquanto em algumas áreas de conhecimento adota-se o inglês, ${ }^{9}$ mesmo para publicações de caráter nitidamente nacional, em busca dessa interlocução, revistas orientadas pela convicção de que uma de suas funções centrais é disseminar materiais na língua nacional parecem condenadas a essa falta de interlocução.

Nesse contexto, a análise de indicadores para avaliação das publicações, a necessidade de visões críticas, impõe-se pelos mais diversos pontos de vista, quer da crítica fundamental que se refere às bases teóricas de tais indicadores, quer quando se introduzem as especificidades da área de investigação. Alertando para a complexidade das análises que tais indicadores merecem Léa Velho ${ }^{10}$ chama a atenção para o quanto, desde os anos de 1980, os fundamentos da tradição mertoniana em sociologia da ciência - que embasam as teorias e estudos empíricos, envolvendo indicadores científicos - têm sofrido inúmeras revisões no contexto das construções críticas dos estudos sociais das ciências. A questão se complica ainda mais quando se busca incorporar perspectivas de gênero em análises de tais indicadores, ou passa-se a construir novos indicadores de sustentação para elaboração de políticas científicas ${ }^{11}$.

\section{Entre desejos e exigências}

No campo dos estudos de gênero, no Brasil, há perspectivas, explicitadas em encontros e seminários, que consideram esse campo como ainda não consolidado. Apesar da ampla produção, há uma argumentação sobre sua fragilidade em termos teóricos e/ ou metodológicos. Discordando dessas leituras, acreditamos que os estudos de gênero, perpassando e envolvendo diversas áreas disciplinares com todas as suas diversidades e especificidades, conformam hoje no Brasil um campo de direito próprio. Isto é evidente não apenas no volume de pesquisas na área, em linhas específicas consagradas nas 
agências de fomento, mas também em seus cursos, congressos e, particularmente, em suas publicações, que garantem visibilidade e acesso ao campo. E também nesse campo, cujas fronteiras são ainda objetos de negociação e construção e, portanto de disputas teóricas, institucionais e políticas, as publicações científicas cumprem sua função básica de articuladoras das diferentes propostas e perspectivas de ação em desenvolvimento.

As publicações acadêmicas que veiculam abordagens feministas estão marcadas pela singularidade de estar orientadas por um interesse político específico. Do nosso ponto de vista, esse interesse é o de compreender, denunciar e oferecer elementos para alterar as maneiras como gênero, articulado a outras categorias de diferenciação, incide no posicionamento desigual das pessoas e, de maneira específica, das mulheres, na vida social. Esse interesse político foi alicerçado no marco de uma série de práticas, tais como o trabalho para desmontar hierarquias em todos os planos possíveis, o que exigia alcançar públicos ou audiências, amplos e diversificados. Todavia, o fato de estarem marcadas por interesses políticos feministas não exime essas revistas da integração num sistema social acadêmico ou ciência.

Nesse sistema social, em um país localizado fora do centro da produção de conhecimento, as publicações acadêmicas feministas estão submetidas a diversas pressões que se entrecruzam tais como: aquelas decorrentes do confronto entre interesses políticos e critérios de excelência acadêmica; a luta pelo posicionamento interno, pois, apesar da legitimidade já obtida no Brasil, essas revistas ainda estão longe de ocuparem os níveis mais altos de estratificação e; finalmente, pela dificuldade na inserção (e valorização) internacional. E, se algumas publicações estão classificadas nos níveis mais elevados concedidos aos periódicos nacionais, no Brasil, acho que muitas concordarão com a idéia de que o impacto e a legitimidade acadêmica obtida por publicar, por exemplo, na Revista Brasileira de Ciências Sociais não é análoga ao de publicar na REF ou nos Cadernos PAGU.

Desde os estudos clássicos de filiação mertoniana da década de 1960, sabemos que, mesmo consideradas as diferentes particularidades das diversas áreas e sub-áreas de estudo não cabe qualquer posicionamento ingênuo quanto ao papel desempenhado pelos "gatekeepers of science". ${ }^{12}$ Nas análises de publicações científicas de qualquer campo disciplinar, os editores das revistas científicas são apontados como os responsáveis por selecionar as informações e pontos de vista em discussão, porque eles jogam um papel decisivo na organização, direcionamento, consolidação e, portanto, controle e definição das fronteiras de acesso à área de estudo. No entanto, os editores precisam articular as demandas de seu corpo editorial, pareceristas, articulistas, público leitor, assinantes, agências de financiamento e avaliadores. Neste processo, as revistas, também na área de gênero, contribuem para a constituição das redes e de 'colégios invisíveis' 13 mantenedores do campo.

É importante perceber, portanto, que nas análises das negociações estabelecidas no âmbito do corpo editorial, isto é, da definição de política das revistas, do sistema de peerreview - da seleção de pareceristas, da aceitação de artigos, etc. - de questões tais como a publicação da data de recebimento e publicação, para que se garanta a prioridade, é necessário considerar o conjunto do sistema e a localização das publicações acadêmicas, inclusive aquelas voltadas para a difusão das abordagens feministas, nesse sistema.

Nesse sentido uma leitura da produção veiculada pelos Cadernos PAGU é interessante, levando em conta alguns pontos que se referem à participação da revista na constituição de um campo de estudos de gênero, no Brasil. A leitura dessa produção permite perceber as dificuldades, contradições, limitações de uma revista que se pretende claramente nacional e que está, em alguns aspectos, na contra-corrente das exigências pela maior inserção internacional das agências de fomento. Isso é explicitado, por 
exemplo, na política de traduções - ponto de honra de revista. Embora não seja um aspecto privilegiado nos sistemas de avaliação no Brasil, a incorporação de traduções é pontuada de maneira especial em modelos de indicadores de produção de países em que se objetiva a consolidação de uma comunidade científica nacional com visibilidade própria e relativo grau de autonomia, em termos internacionais.

Valorar de formas diferenciadas indicadores específicos, não significa particularmente nesse caso, ignorar que a captação Núcleo de Estudos de Gênero-PAGU/UNICAMP altamente desejável -, de artigos inéditos de pesquisadores estrangeiros resulta de um processo de inserção e reconhecimento da veiculação internacional da publicação. Nesse caminho, no que se refere à veiculação da produção internacional nos Cadernos, esta reflete em certa medida cooperações internacionais, formações acadêmicas, quadros referenciais, preferências teóricas. Revela uma concentração de trabalhos de autoras/es sediados nos Estados Unidos e na Europa, particularmente, na França, Inglaterra e Portugal, e baixa participação de colaboradores da América Latina, que são precisamente aqueles que, em diversos sentidos, compartilham tanto uma realidade social mais próxima, como uma proximidade lingüística.

No que se refere ao conteúdo, a revista mostra a tentativa de articular a difusão de inquietações, reflexões e pesquisas produzidas no âmbito internacional com a análise de problemáticas relevantes na realidade nacional. Mas, nesse ponto, não se diferencia necessariamente dos esforços realizados por diversas revistas da área de ciências sociais. ${ }^{14} \mathrm{~A}$ diversidade disciplinar presente na revista é relativamente restrita e mantém relações com as vinculações disciplinares dos integrantes do comitê editorial. ${ }^{15}$ No que se refere à inserção institucional dos colaboradores, os Cadernos Pagu, apesar de amplas iniciativas de captação de material, apresentam uma concentração de autoras e autores vinculados a instituições do Rio e São Paulo, que tendem a ser consideradas as mais prestigiosas do país.

Este indicador - filiação institucional dos autores - também não foge ao padrão analisado desde os estudos clássicos de Diana $\mathrm{Crane}^{16}$, hoje recorrentes, reiterados inclusive por diversos estudos latino-americanos e, sem dúvida, acompanha o conjunto da concentração da produção científica nas diversas áreas disciplinares no país ${ }^{17}$. Para aquela autora, o reconhecimento da produção de um cientista tende a estar vinculado ao prestígio associado às principais universidades, e o sistema da avaliação anônima, isto é, avaliações nas quais o avaliador não tem acesso ao nome e à vinculação institucional do autor, não garante a incorporação de autores de universidades menos prestigiosas nas publicações. A superação de tais contradições relaciona-se menos a esforços localizados de editores, na tentativa de cumprir exigências de indicadores artificiais e mais, a políticas institucionais de longo prazo, de incentivo à pesquisa e publicação, bem como à ampliação ainda maior do campo disciplinar.

Chamamos a atenção para esses pontos, e ainda de uma maneira exploratória, não com o interesse de fazer uma leitura crítica da revista, mas porque eles mostram que os Cadernos não escapam aos mecanismos de constituição de um campo de conhecimentos, nos quais as revistas acadêmicas adquirem centralidade. Em função das tensões e pressões às quais estão submetidas as revistas acadêmicas que estão "nas margens", a , em termos políticos e teóricos, vinculadas ao movimento feminista não tem produzido necessariamente uma inovação análoga no que se refere à dinâmica e estabelecimento das relações que constituem os campos de estudo.

\section{Concluindo: a constituição de um campo de estudos}

Concluindo, é importante sublinhar que esta análise não é necessariamente negativa. Precisamente, a adequação à lógica de constituição desses campos têm 
possibilitado a crescente legitimidade do campo de estudos de gênero no país, contribuindo para a criação de cursos, particularmente nas pós-graduações. E esses cursos têm viabilizado a formação de feministas, inclusive de feministas não necessariamente voltadas para a ałuação no âmbito acadêmico. No entanto, esta leitura oferece uma excelente oportunidade para se pensar sobre os acertos e as limitações dessas revistas acadêmicas, refletindo sobre os elementos que podem ser incorporados nas negociações às quais elas não podem escapar.

Cabe perguntar quais são os mecanismos que seriam necessários ativar para ver nesses periódicos, além de conteúdos que deixem claro o caráter político das relações perpassadas por gênero, em toda sua complexidade, uma maior diversificação, como a presença de um número maior de jovens autores, vinculados a espaços institucionais afastados dos centros desta 'periferia'. Como bem explica Lea Velho o peer review "baseiase em desempenho passado para prever desempenho futuro. Conseqüentemente, existem barreiras significativas à entrada de novos participantes, o que provoca a formação de uma elite científica que controla o sistema de comunicação, de recompensa e de alocação de propriedades na ciência. Tal fenômeno foi reconhecido até mesmo por Merton - 'o efeito Matheus" 18 , e tem sido amplamente criticado em níveis científicos e políticos internacionais desde os anos de $1970^{19}$. Como o campo de estudos tem-se posicionado frente a essas não tão novas questões? Cabe perguntar também como viabilizar que nossa produção conte com uma interlocução internacional, com repostas "de fora", sobretudo como construir uma interlocução que integre não apenas os países da América Latina, mas também as outras nações do Sul, na África e Ásia. Cabe, enfim, pensar nos caminhos a serem seguidos, mas sem ingenuidade, conscientes da maneira como a lógica que incide na organização dos campos de estudo afeta as publicações acadêmicas, inclusive as feministas.

\section{Notas}

Copyright @ 2004 by Revista Estudos Feministas.

${ }^{1}$ As autoras agradecem à Profa. Dra. Maria Conceição da Costa e à doutoranda Cristina Escobar (DPCT-IG/ UNICAMP) pelas sugestões de referências bibliográficas e a lara Beleli por sua leitura atenta do texto.

${ }^{2}$ Hebe VESSURI, 1987, p. 124. Esta afirmação se apóia nos escritos de Derek de Solla Price (1969), nos quais ele já definia o cientista como aquele que publica, como papirófilo em oposição ao tecnólogo papirofóbico. ${ }^{3}$ Lea VELHO, 1997.

${ }^{4}$ VESSURI, 1987, p. 124; VELHO, 1997, p. 16.

${ }^{5}$ PRICE, 1963.

${ }^{6}$ Elea GIMÉNEZ TOLEDO y Adelaida R. ROMÁN, 2000.

7 VESSURI, 1987, p. 124

${ }^{8}$ VESSURI, 1987, p. 126

${ }^{9}$ Sobre o publicar em inglês, desde o início dos anos 80, quando essa discussão sobre indicadores ganhou força na América Latina, Roche y Freites, (1982), já destacavam que a busca de suas razões não estava na existência de qualquer complô obscuro que nos obrigasse a publicar em inglês, mas sim nos processos históricos, associados indubitavelmente a hegemonias políticas, sustentadas por desenvolvimentos científicotecnológicos prodigiosos dos países de língua inglesa. Os autores lembram também que o monopólio de determinado idioma na comunicação científica mundial não é um fenômeno recente, mas pelo contrário é um fenômeno cambiante a cada contexto histórico específico.

${ }^{10}$ VELHO, 2001.

11 LOPES, 2002

12 Diana CRANE, 1967.

${ }^{13}$ CRANE, 1972

14 VELHO, 1997

${ }^{15}$ No que se refere às disciplinas nas quais se ancora a produção veiculada na publicação há uma concentração nas áreas de antropologia (32\%), história (23\%), sociologia (21\%), teoria literária e lingüística 
(9\%), filosofia (4\%), educação (3\%), psicologia (2\%). Outras áreas disciplinares estão presentes apenas com $1 \%$ da produção - biologia, geografia, informática, jornalismo, ciência política, medicina, química.

${ }^{16}$ CRANE, 1972.

17 FAPESP, 2000

${ }^{18}$ VELHO, 1997, p. 112. Para uma crítica irônica, muito bem humorada, mas mordaz sobre tais mecanismos que contribuem para a invisibilidade das mulheres no conjunto da produção científica ver Margaret ROSSITER (1993)

19 Daryn CHUBIN e Edward HACKETT, 1990.

\section{Referências}

CHUBIN, Daryn; HACKETT, Edward. Peerless Science, Peer Review and U.S. Science Policy. Albany: State University of New York, 1990.

CRANE, Diana. Invisible Colleges. Diffusion of knowledge in Scientific Communities. Chicago \& London: Chicago University Press., 1972.

CRANE, Diana. "The gatekeepers of science, some factors affecting the selection of articles for scientific journals". The American Sociologist. p. 195-201, Nov. 1967.

GilMÉNEZ-TOLEDO, Elea; ROMÁN-ROMÁN, Adelaida. "Evaluación de revistas científicas: análisis comparativo de dos modelos y su aaplicación a cinco revistas españolas de biblioteconomía y documentación". INTERCIENCIA, v. 25 n. 5, p. 234-41, Aug. 2000.

LOPES, Maria Margaret. "As grandes ausentes das inovações tecnológicas em ciência e tecnologia". Resenha do livro: TABAK, Fanny. O Laboratório de Pandora. Cadernos Pagu, v. 19, p. 315-18, 2002.

PRICE, J. Derek de Solla. Little Science, Big Science. New York: Columbia University Press., 1963.

PRICE, J. Derek de Solla. "Measuring the size of science". In: Proceedings of the Israel Academy of Sciences and Humanities, v. 4, p. 98-106, 1969.

ROCHE, Marcel; FREITES, Yajara. "Producción y Flujo de información científica en un país periférico Americano (Venezuela)". INTERCIENCIA, v. 7, n.5, p.279-90, sep-oct. 1982.

ROSSITER, Margaret W. "The Mathew-Matilda effect in science". Social Studies of Science, v. 23, p. 325-41, 1993.

VELHO, Lea M. S. "Estratégias para um sistema de indicadores de C \& T no Brasil". Parcerias Estratégicas, v.13. Brasília: Ministério da Ciência e Tecnologia, p.109-21, dez. 2001.

VELHO, Lea M. S. "A ciência e seu público". Transinformação, v. 9, n. 3, p. 16-32, set./dez. 1997.

VESSURI, Hebe M. C. "La Revista Científica Periférica. El caso de Acta Científica Venezolana". INTERCIENCIA, may-june, v. 12, n. 3, p. 124-34, 1987.

\section{Scientific Journals and The Construction of "Gender Studies": a "Peripheral" Look}

Abstract: This paper deals with the role of scientific journals on the constitution of the field of gender studies in a peripheral country, Brazil. Studies in sociology of science, the role played in the fields of history of science and R\&D policy by scientific papers and the author's experience as editors of CADERNOS PAGU constitute the tools to analyze the interaction of publishing and feminist agency in the production of knowledge. Our aim is to discuss the effect of tensions and "negotiations" in the publication of scientific journals and in the constitution of the field of gender studies. The political agency, tensions, "negotiations", peer-review processes, "gate-keeping" of scientific knowledge, editorial and translation policies and so on are dealt with in the framework of science publication, the "speciation" of a field of research and the reproduction of centrality and periphery within our own country. Finally, we pointed to directions to increase journal visibility, content diversification and to foster cooperation with Latin American and international researchers. Keywords: gender studies, history of science, scientifics reviews, feminism. 